\title{
La promoción de valores relacionados con los Objetivos de Desarrollo Sostenible (ODS) en la programación de Playz
}

\section{Promotion of values related to Sustainable Development Goals (SDG) in Playz's programming}

\begin{abstract}
Isaac Maroto González - Universidad de Santiago de Compostela isaac.maroto@rai.usc.es
\end{abstract}

Talía Rodríguez Martelo - Universidad Complutense de Madrid talrodri@ucm.es

Resumen: Desde el año 2016 los Objetivos de Desarrollo Sostenible han marcado la ruta política y social de los países que adquirieron el compromiso de la agenda 2030. Dichos objetivos promueven valores de protección hacia las personas, la sociedad y el medio ambiente y forman parte de la estrategia de responsabilidad social de la corporación de RTVE. A través de su canal Playz, cuyo público principal son los jóvenes, RTVE articula una serie de contenidos audiovisuales que atienden a múltiples temáticas. En calidad de canal perteneciente a la televisión pública, este trabajo analiza la función de servicio público en la difusión concreta de la información y los valores expuestos en su estrategia de comunicación y responsabilidad social corporativa. La investigación planteada propone una aproximación analítica y 


\section{Las nuevas narrativas, en el entorno social \\ Universidad de La Laguna, diciembre de 2019}

descriptiva del canal Playz y cómo integra la divulgación y promoción de los valores de los ODS a través de sus contenidos audiovisuales y nuevos formatos interactivos.

Palabras clave: ODS, Playz, televisión, servicio público, valores

Abstract: Since 2016, the Sustainable Development Goals have marked the political and social route of the countries that acquired the 2030 agenda commitment. These objectives promote protection values towards people, society and the environment and RTVE has made them part of the strategy of its social responsibility corporation. Through its Playz channel, whose main audience is young people, RTVE articulates a kind of audiovisual content that address multiple subjects. As a channel belonging to public television, this work analyzes the public service function in the specific dissemination of information and the values exposed in its communication strategy and social responsibility. The research proposes an analytical and descriptive approach to the Playz channel and how it integrates the dissemination and promotion of the SDG values through its audiovisual content and new interactive formats.

Key words: SDG, Playz, television, public service, values,

\section{Introducción}

El medio televisivo facilita la atención e interés respecto a otros medios de comunicación clásicos como la prensa debido a su naturaleza audiovisual. La capacidad tecnológica de este medio posibilita una amplia difusión territorial y continua de información diaria que lo han convertido durante décadas en la pantalla líder a la hora de aglutinar la audiencia de diversos públicos. Este hecho, convierte el medio televisivo en una herramienta de constructo social ya que los espectadores, mediante el visionado de los contenidos televisivos consiguen ampliar la experiencia y el conocimiento del mundo, más allá de las experiencias que perciben en su vida cotidiana. (Fishman, 1980) 


\section{Las nuevas narrativas, en el entorno social}

Universidad de La Laguna, diciembre de 2019

A pesar de que la penetración de Internet en los hogares españoles continúa creciendo (hasta un $77,9 \%$ en 2018), la televisión continúa siendo la pantalla favorita con el mayor índice de consumo (unos 210 minutos al día.) ${ }^{1}$ Sin embargo, lo que sí ha cambiado es este consumo que se disgrega a través de varias pantallas o el fenómeno multitasking consistente en la visión simultánea de contenidos audiovisuales en televisión y pantalla móvil. En cualquier caso, el efecto divulgador ya sea en una o varias pantallas aporta información de actualidad a las diferentes audiencias.

Esta capacidad de aprehensión del conocimiento que aporta la televisión, la condiciona directa o indirectamente como una herramienta de aprendizaje que influye en la formación de los espectadores, en especial del público infantil y adolescente:

La televisión influye en todas las edades pero de manera especial en la infancia y la adolescencia, la parte más vulnerable del numeroso público expuesto, dada su incompleta formación ideológica, social, cultural y de valores. Sin embargo, se ha dejado sentir también en la vida cotidiana de los adultos. (Rodríguez Escámez, A. 2005 p. 3).

La influencia que puede ejercer en la sociedad el sector televisivo a través de sus contenidos como medio de comunicación masivo, lo determinan como un ente empresarial de gran influencia social al que se le exige una Responsabilidad Social igual o mayor que a cualquier otra empresa o sector. (Sierra, y Pascual, 2012)

\section{Marco teórico y contextual: la responsabilidad social corporativa de la televisión pública}

\section{Los objetivos de la televisión pública}




\section{Las nuevas narrativas, en el entorno social Universidad de La Laguna, diciembre de 2019}

La potencia persuasiva de la televisión llama la atención de los gobiernos desde sus orígenes lo que da lugar al desarrollo de una televisión pública al servicio de la sociedad. El medio televisivo surge como un impulso que potencia el desarrollo y evolución de la población a través de unos objetivos y funciones específicas: educar, informar y entretener, la Unión Europea de Radiodifusión amplía y matiza esos objetivos transformándolos en seis valores: independencia, universalidad, diversidad, calidad, innovación y rendición de cuentas. (EBU, 2014)

La televisión pública como un elemento constitutivo de la sociedad al servicio público debe de emanar valor social, entendiendo este valor como una cualidad y estimación que se le otorga a las instituciones a través de categorías objetivas y subjetivas donde se combinan los valores sociales, valores individuales, valores de cambio y funcionalidad del medio televisivo. Como elemento de servicio público institucional conlleva el valor político de la televisión pública con el fin de defender valores constitucionales, democráticos y plurales que promuevan la cohesión social a partir del desarrollo y fomento de la diversidad en su población, atendiendo a la protección de grupos vulnerables y alfabetización mediática de la sociedad. (Campos Freire, 2017).

Tal y como se muestra en la página corporativa de RTVE, la televisión española se autodefine como referente de calidad, garantizando la pluralidad de programación, diversa y equilibrada para diferentes públicos, y de esta forma ofrecer información veraz que ayude a fomentar el debate, ofrecer contenidos educativos y de entrenamiento de calidad. A su vez destaca su compromiso tecnológico adaptándose al nuevo escenario digital, así como al desarrollo sostenible.

La televisión pública en relación a los criterios de programación televisiva destaca por su oferta de contenidos equilibrados que respondan a las diferentes necesidades demandadas por la sociedad donde predomina la 


\section{Las nuevas narrativas, en el entorno social Universidad de La Laguna, diciembre de 2019}

planificación cultural en diseño de parrillas que ofrezca un equilibrio entre diferentes géneros. Todo en concordancia con la rentabilidad de dichos contenidos y poniendo énfasis a en ofrecer contenidos a minorías como público televisivo, liberándose así de la atadura de satisfacer a una audiencia mayoritaria como es el caso de la televisión comercial. (Izquierdo, 2016).

\section{Los objetivos de desarrollo sostenible y su función}

En el año 2000 se plantearon los Objetivos del Milenio (ODM), una iniciativa de la ONU para dar forma a un mundo más igualitario, justo y sostenible para todas las naciones. La estrategia planteada llegaba hasta el año 2015. En esta fecha entraba en vigor una nueva hoja de ruta que recogía el testigo de estos objetivos y se ampliaban para conformar los denominados Objetivos de Desarrollo Sostenible (ODS). (Objetivos de desarrollo sostenible, 2019)

El 25 de septiembre de 2015, los líderes mundiales adoptaron un conjunto de objetivos globales para erradicar la pobreza, proteger el planeta y asegurar la prosperidad para todos como parte de una nueva agenda de desarrollo sostenible. Cada objetivo tiene metas específicas que deben alcanzarse en los próximo 15 años. (Objetivos y metas de desarrollo sostenible, UN, 2019)

De esta forma se establecían las directrices centradas en la consecución de 17 objetivos concretos y 169 metas propuestas por los 193 estados miembros de la ONU que aprobaron en dicha cumbre la agenda 2030 titulada: Transformar nuestro mundo: la agenda 2030 para el desarrollo sostenible. (Objetivos de desarrollo sostenible, 2019)

Los objetivos que recoge esta agenda y que dotan de contenido a los ODS son los siguientes: 


\section{Las nuevas narrativas, en el entorno social \\ Universidad de La Laguna, diciembre de 2019}

Objetivo 1: Poner fin a la pobreza en todas sus formas en todo el mundo

Objetivo 2: Poner fin al hambre, lograr la seguridad alimentaria y la mejora de la nutrición y promover la agricultura sostenible

Objetivo 3: Garantizar una vida sana y promover el bienestar para todos en todas las edades

Objetivo 4: Garantizar una educación inclusiva, equitativa y de calidad y promover oportunidades de aprendizaje durante toda la vida para todos

Objetivo 5: Lograr la igualdad entre los géneros y empoderar a todas las mujeres y las niñas

Objetivo 6: Garantizar la disponibilidad de agua y su gestión sostenible y el saneamiento para todos

Objetivo 7: Garantizar el acceso a una energía asequible, segura, sostenible y moderna para todos

Objetivo 8: Promover el crecimiento económico sostenido, inclusivo y sostenible, el empleo pleno y productivo y el trabajo decente para todos

Objetivo 9: Construir infraestructuras resilentes, promover la industrialización inclusiva y sostenible y fomentar la innovación

Objetivo 10: Reducir la desigualdad en y entre los países

Objetivo 12: Garantizar modalidades de consumo y producción sostenibles

Objetivo 13: Adoptar medidas urgentes para combatir el cambio climático y 
sus efectos

Objetivo 14: Conservar y utilizar en forma sostenible los océanos, los mares y los recursos marinos para el desarrollo sostenible

Objetivo 15: Proteger, restablecer y promover el uso sostenible de los ecosistemas terrestres, gestionar los bosques de forma sostenible, luchar contra la desertificación, detener e invertir la degradación de las tierras y poner freno a la pérdida de la diversidad biológica

Objetivo 16: Promover sociedades pacíficas e inclusivas para el desarrollo sostenible, facilitar el acceso a la justicia para todos y crear instituciones eficaces, responsables e inclusivas a todos los niveles

Objetivo 17: Fortalecer los medios de ejecución y revitalizar la Alianza Mundial para el Desarrollo Sostenible
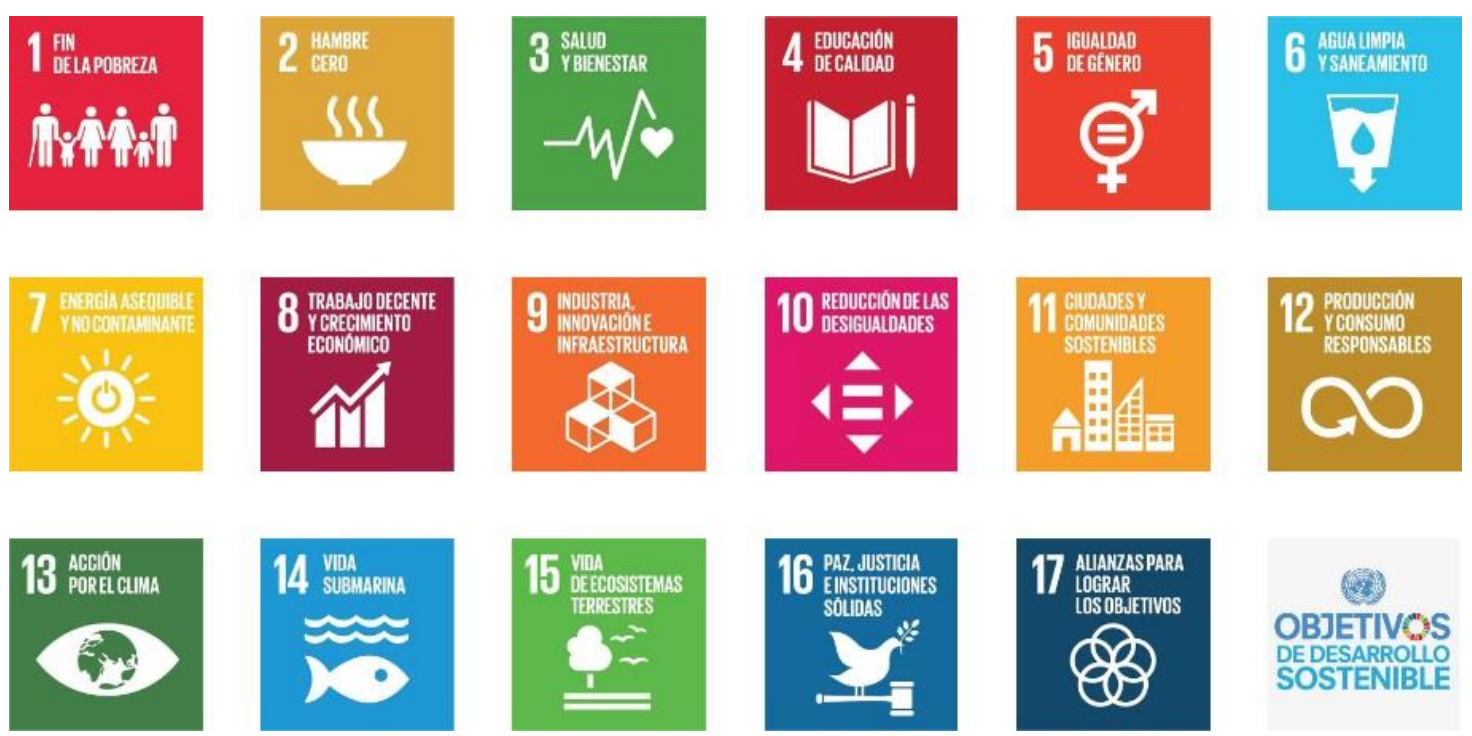

Ilustración 1. Fuente: Objetivos y metas de desarrollo sostenible, UN (2019) https://www.un.org/sustainabledevelopment/es/objetivos-de-desarrollo-sostenible/ 


\section{Las nuevas narrativas, en el entorno social \\ Universidad de La Laguna, diciembre de 2019}

\section{RTVE y los ODS}

RTVE como empresa de servicio público en su memoria de RSC de 2017 refleja su compromiso como firmante en el Pacto Mundial y manifiesta su intención de trabajar en la implantación de los 17 retos de los Objetivos de Desarrollo Sostenible (ODS). De esta forma, todas las marcas que engloban la corporación (RTVE, TVE, RNE, RTVE.ES) utilizan su capacidad como medio de comunicación para ampliar y difundir los 17 objetivos que permitan acercarse a las 169 metas para proteger el futuro colectivo en las diferentes áreas que concretan los ODS.

Para establecer la prioridad de los ODS en función del orden de actuación, la corporación se tuvo en cuenta tanto en las preferencias del propio negocio de la corporación como las preferencias de los grupos de interés. Para conocer las particularidades de los grupos de interés se obtuvo información a través de diferentes fuentes como: los foros celebrados por la Corporación, la recopilación y análisis de las noticias relacionadas con los diferentes aspectos de los ODS, y el estudio de las tendencias del sector audiovisual a través de informes publicados por los otros medios de comunicación del sector.

Por otro lado, las directrices que marcan las prioridades por parte de la Corporación de RTVE establecen las exigencias de negocio por parte del canal público de televisión. Estas directrices se toman a partir de las declaraciones del presidente ${ }^{2}$ así como de miembros del Comité de Dirección de RTVE y son completadas con datos extraídos de las Memorias del servicio público de RTVE.

A partir de las variables establecidas en los puntos anteriores RTVE desarrolla un esquema que refleja el nivel de interés en los diferentes Objetivos de Desarrollo Sostenible en el que deja constancia en la Memoria de la Corporación de 2017. 


\section{Las nuevas narrativas, en el entorno social \\ Universidad de La Laguna, diciembre de 2019}

Matriz de asuntos relevantes para RTVE 2017 (1)

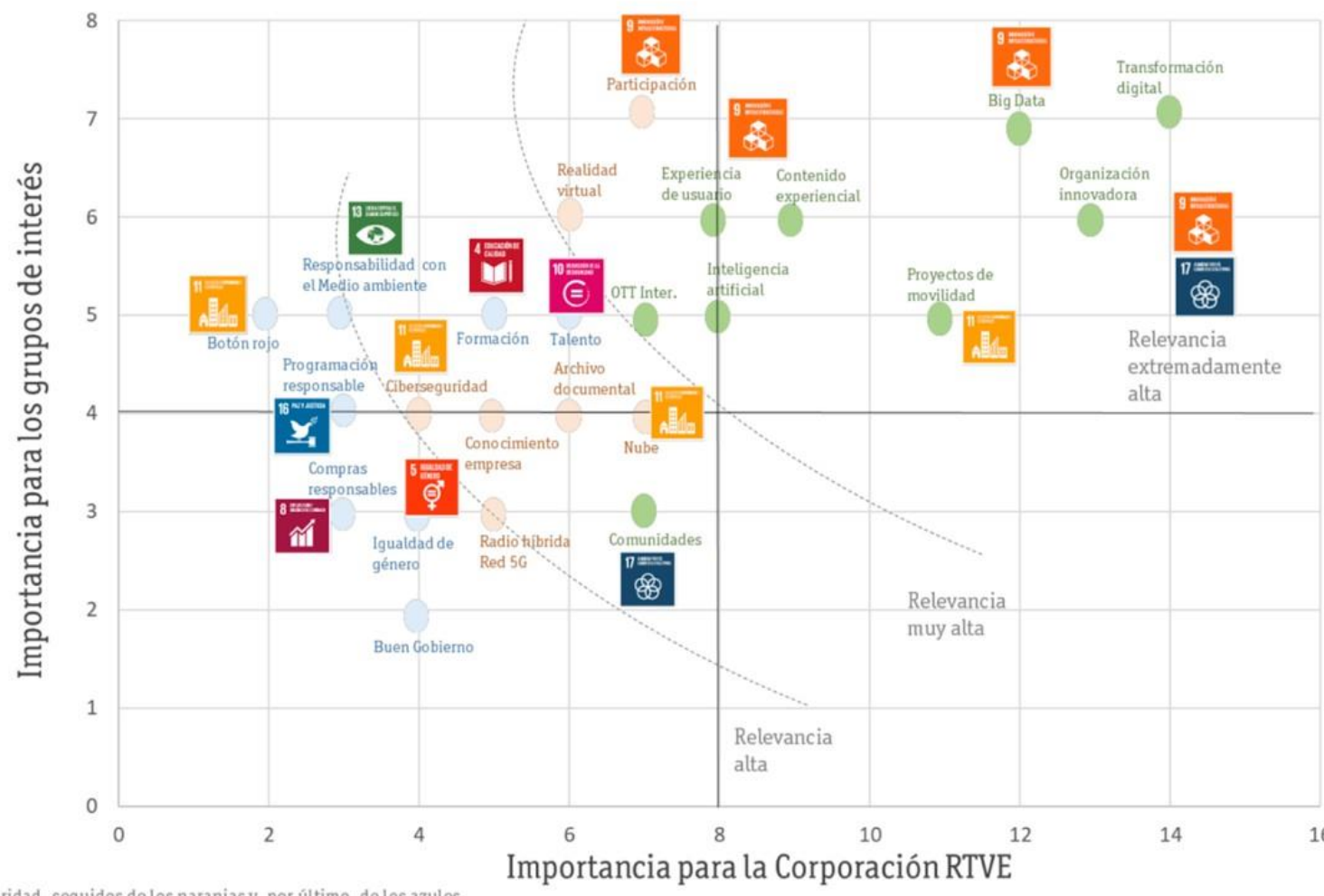

Core Business RTVE

Ilustración 2. Fuente: Dossier de RSC de RTVE (2018), recuperado el 5 de noviembre en www.rtve.es

En la matriz de asuntos relevantes destacan los objetivos de la Corporación televisiva y cómo éstos están relacionados con algunos aspectos de desarrollo tecnológico que se encuentran en relación con los Objetivos de Desarrollo Sostenible. En estos aspectos confluyen la transformación digital y la implantación de la tecnología Big Data junto con el contenido experiencial que está vinculado a las demandas de los nuevos públicos.

En definitiva, la matriz refleja que el esfuerzo o interés por priorizar los ODS se concentra en los objetivos que coinciden con los objetivos corporativos de negocio. De esta forma, el esfuerzo por parte de RTVE deja relegados a menor interés o menor importancia las propias demandas de los denominados "grupos de interés" como pueden ser la responsabilidad con el medio ambiente, 


\section{Las nuevas narrativas, en el entorno social \\ Universidad de La Laguna, diciembre de 2019}

la igualdad de género, la igualdad de oportunidades o el buen gobierno, hecho destacable teniendo en cuenta la naturaleza pública de la propia corporación televisiva

\section{Metodología}

El marco metodológico de la investigación se desarrolla a partir de una perspectiva descriptiva y cualitativa. Atendiendo a este enfoque y desde una aproximación al análisis de contenido de la programación de Playz, analizaremos la relación entre la programación de la plataforma y la divulgación directa e indirecta de los Objetivos de Desarrollo Sostenible.

En este sentido, se han establecido dos campos de análisis diferenciados. En primer lugar, de forma genérica se ha confrontado la matriz de análisis sobre los valores de los ODS que la corporación RTVE ha marcado dentro de su línea estratégica con los que inciden en la parrilla de contenidos de Playz. En segundo lugar, se ha realizado un visionado de contenidos estimando los ODS más relevantes aludidos en cada contenido en particular.

Dado que la plataforma Playz tiene un sistema de emisión de contenidos expansiva, hemos situado el análisis de la programación conforme a los mayores índices de difusión y presencia en el período seleccionado entre el 4 y el 10 de noviembre de 2019. "A diferencia del clásico análisis de contenido, que principalmente consiste en registrar meras ocurrencias o frecuencias en un producto mediático (y por lo tanto es cuantitativo por definición), el análisis de contenido cualitativo se basa en la construcción y codificación sistemáticas de categorías, no necesariamente pre-establecidas." (Tortajada y Willem, 2019) Partiendo de esta definición metodológica, en este caso, seleccionamos las variables a partir de categorías predefinidas, por lo tanto, el análisis cualitativo está basado en la identificación positiva o negativa de los ODS sobre los contenidos clasificados. 


\section{Las nuevas narrativas, en el entorno social \\ Universidad de La Laguna, diciembre de 2019}

\section{Discusión y resultados}

Estableciendo un paralelismo con la matriz de asuntos relevantes para RTVE que pone en relación los ODS con los grupos de interés y la corporación, realizaremos la misma evaluación sobre la oferta de contenidos de Playz, así como el canal Playz en su totalidad entendiendo qué supone el proyecto dentro de la estrategia de RTVE.

Hemos de tener en cuenta la función desarrollada por el medio televisivo y cómo este servicio hacia el espectador está íntimamente relacionado con su capacidad de transmisión de entretenimiento y conocimiento, ya que "tenemos que afirmar que la televisión en su conjunto realiza una labor educativa en positivo o en negativo". (García Matilla, 2003)

En este sentido, los valores que pueden ser relacionados con cada contenido de Playz, así como los que se transmiten a partir de la configuración de la parrilla de la plataforma digital, se han analizado tratando de establecer si están en consonancia con los valores declarados por RTVE y si es equiparable a la matriz de interés sobre los Objetivos de Desarrollo Sostenible. Tal y como se recoge en Fabbro y Sánchez-Labella (2016), la infancia y la juventud están, cada vez más, integrados en entornos de aprendizaje informal que en gran medida es transmitido a través de los contenidos audiovisuales recogidos en televisión y plataformas digitales, es por ello que resulta crucial analizar y entender qué valores son difundidos en el contexto de la televisión pública.

La parrilla de contenidos de Playz tiene una estructura expansiva y con tendencia a la navegación circular en contraposición a las estructuras lineales clásicas. Tal y como se ha indicado en la metodología, los contenidos analizados se corresponden a los destacados durante el período de análisis entre el 4 y el 10 de noviembre, y se ha centrados en los contenidos no ficcionados. En la plataforma destinada a público joven (millenials y Generación Z), existe una gran profusión de contenidos de género documental con 
diferentes formatos que abarcan desde planteamientos interactivos a documentales seriados.

A continuación, se puede observar una relación de contenidos analizados indicando el género y temática principal y los Objetivos de Desarrollo Sostenible identificados a partir de la visualización realizada. Como ya avanzamos en trabajos anteriores (Maroto González y Rodríguez Martelo, 2018), los géneros de los contenidos de la plataforma no responden a modelos clásicos, de forma que la identificación de los mismos ha sido realizada desde una aproximación descriptiva más que desde una intención de clasificación formal:

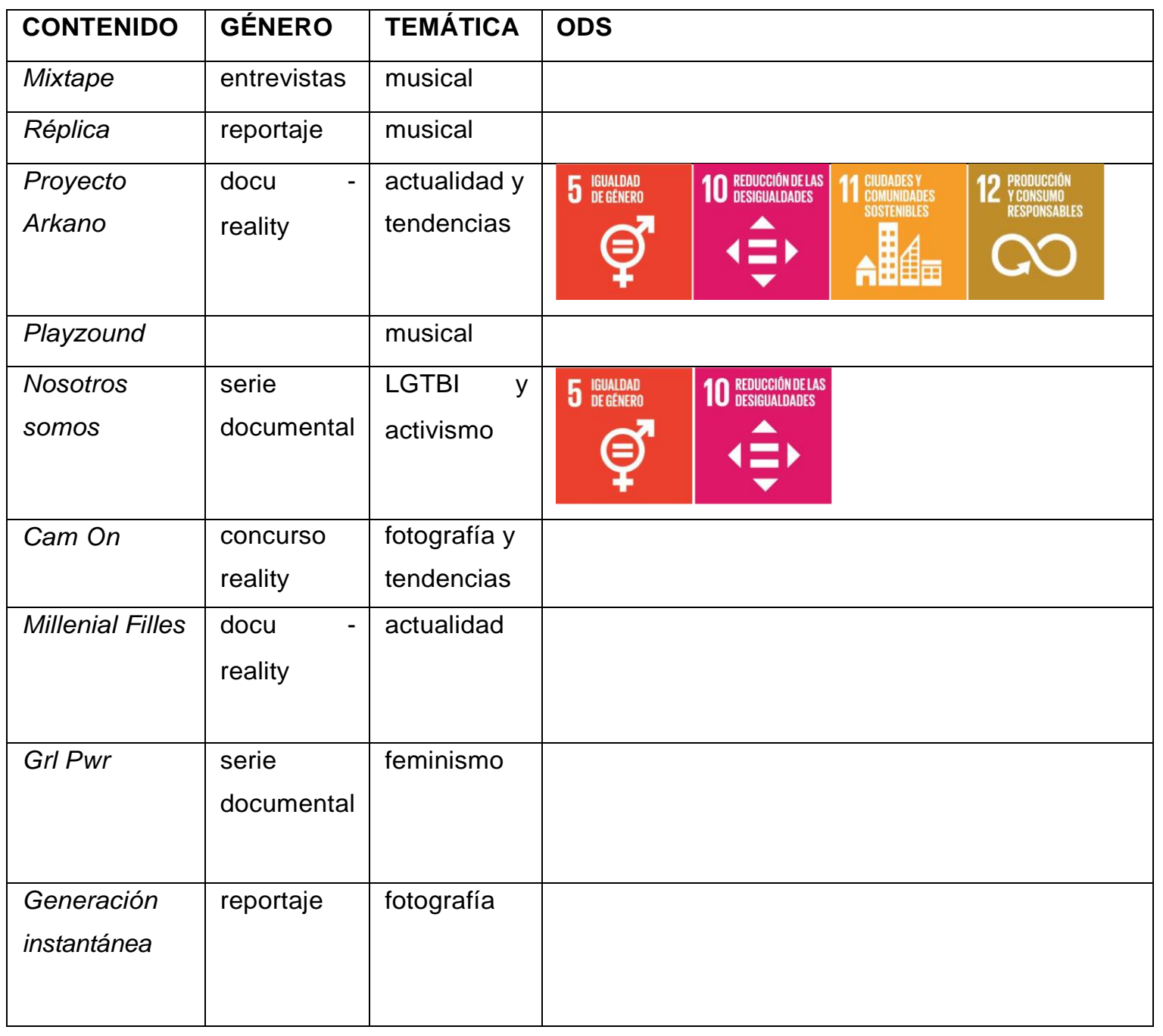




\section{Las nuevas narrativas, en el entorno social}

Universidad de La Laguna, diciembre de 2019

\begin{tabular}{|c|c|c|c|c|c|c|c|}
\hline En la brecha & $\begin{array}{l}\text { documental } \\
\text { interactivo }\end{array}$ & feminismo & 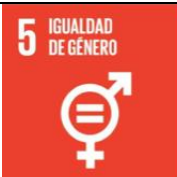 & 10 REDUCEÓNDELLAS & & & \\
\hline Binario & documental & $\begin{array}{l}\text { actualidad, } \\
\text { debate }\end{array}$ & 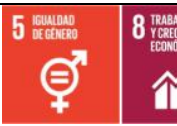 & 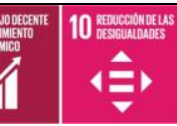 & A: & $\begin{array}{c}12 \text {. } \\
90\end{array}$ & 13 \\
\hline $\begin{array}{l}\text { Guerra a la } \\
\text { mentira }\end{array}$ & $\begin{array}{l}\text { documental } \\
\text { interactivo }\end{array}$ & actualidad & 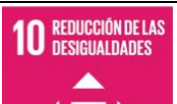 & 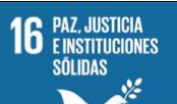 & & & \\
\hline Sin huella & $\begin{array}{l}\text { documental } \\
\text { interactivo }\end{array}$ & $\begin{array}{ll}\text { ecología y } \\
\text { cambo } \\
\text { climático }\end{array}$ & 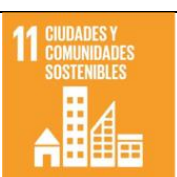 & 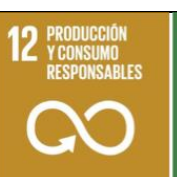 & 13 PORELCLIM & & \\
\hline
\end{tabular}

Tabla 1. Elaboración propia.

En la matriz de temas de interés de valores compartida entre la corporación RTVE y sus públicos predominan los siguientes objetivos:

Encontramos una mayor presencia de los tres primeros $(9,11$ y 17$)$ y en menor medida los objetivos número 4, 5, 10, 13 y 16.

La correspondencia entre los intereses de RVTE con los ODS y su inferencia en la estrategia de la corporación se centra en la innovación en las infraestructuras, la sostenibilidad y las alianzas. También observamos una predisposición hacia otros ODS como la promoción de la igualdad, la igualdad de género y la protección del medio ambiente como ejes transversales. Es en este punto en el que la matriz de RTVE y los ODS representados de forma predominante en los contenidos de Playz coinciden.

La parrilla de Playz tiene una amplia variedad de temáticas, pero comparten de forma visible la preferencia por la difusión y divulgación de contenidos culturales, ya que existe una profusión de contenidos documentales que apelan a la cultura y a la actualidad a través de diferentes formatos. 
En los programas de género musical no se ha identificado ningún ODS concreto de la lista de la agenda 2030, pero si se observa una intención de promoción de la cultura que se podría enlazar de forma genérica con la educación de calidad. Los valores predominantes que destacamos una vez realizado el análisis son los que apelan a promoción de la igualdad e igualdad de género destacando la temática feminista como ODS con mayor constancia representado en diversos contenidos como Nosotros Somos, Grl Pwr, En la Brecha o varios programas de Proyecto Arkano. De igual forma se enfatizan y se debaten desde una perspectiva informativa los ODS que se relacionan con el trabajo digno, la sostenibilidad, la producción y el consumo responsables y

la acción por el clima, teniendo estos valores contenidos exclusivos dedicados como Sin Huella, diversos capítulos de Binario o Millenial Files que alude a estas temáticas en clave de humor. Los nuevos formatos que se desarrollan en la plataforma confieren un compromiso con la innovación, ODS que trabaja activamente la corporación RTVE, así como la promoción de la paz y las alianzas estratégicas, valores a los que Playz dedica el documental interactivo Guerra a la mentira.

\section{Conclusiones y recomendaciones.}

La plataforma digital Playz constituye la apuesta estratégica y el compromiso de RTVE en el desarrollo de contenidos audiovisuales innovadores y centrados en las nuevas audiencias jóvenes. De esta forma, Playz trabaja con nuevos formatos adaptándose a las nuevas tendencias y temáticas del público millenial y la Generación Z y con una clara vocación de divulgación cultural y nuevos valores sociales asociados a las tendencias actuales y nuevas generaciones de consumidores.

Como organismos de servicio público, RTVE y su plataforma Playz participan de las estrategias internacionales para la agenda 2030 cuyo contenido se materializa a través de los 17 Objetivos de Desarrollo Sostenible. Los intereses de RTVE y de Playz vinculados a estos objetivos difieren 


\section{Las nuevas narrativas, en el entorno social Universidad de La Laguna, diciembre de 2019}

sensiblemente debido a la perspectiva empresarial de RTVE orientada a la innovación y la infraestructura como valores destacados, mientras en Playz destacan los valores de promoción de la igualdad y la acción por el clima y el consumo sostenible a través de una profusión de contenidos de índole social.

En definitiva, en la plataforma Playz, la corporación de RTVE ha buscado ofrecer en sus contenidos unas temáticas actuales, cercanas a las tendencias e intereses que refleja el público millenial y, a su vez, coincide con algunos aspectos que se persiguen en los ODS más cercanos al carácter social, de igualdad y medio ambiente y menos centrados en los objetivos empresariales.

Cabe destacar que en la creación de la plataforma confluyen dos intereses que en sí mismos son complementarios desde diferentes perspectivas: El interés por favorecer el desarrollo educativo de la sociedad y la alfabetización mediática a través de contenidos de calidad, un objetivo propio de una televisión pública del que emana el valor social del servicio público televisivo. A su vez, los ODS en su objetivo numero cuatro en el que búsqueda favorecer la educación de calidad a través de una educación inclusiva y equitativa se encuentra en relación con los objetivos de la televisión pública que se expanden gracias a la internacionalización de sus contenidos en Latinoamérica a través de Internet.

En comparación con los objetivos de desarrollo marcados en la memoria de RTVE en 2017, dos años después, esta investigación trata de mostrar que sus preferencias se han modificado, ya sea por el peso de las demandas de los nuevos públicos o por un interés marcado por la propia corporación televisiva, datos que se podrán corroborar con la próxima memoria corporativa de RTVE española.

En general, el camino emprendido con la plataforma digital Playz viene a constituir una oferta de contenidos que tratan de aunar actualidad y valores 


\section{Las nuevas narrativas, en el entorno social \\ Universidad de La Laguna, diciembre de 2019}

sociales dentro de los cuáles podemos identificar muchos de los ODS de la agenda 2030, y desde este punto de vista, el trabajo conjunto resulta pertinente y atractivo como pauta para el desarrollo de una programación televisiva de servicio público pero adaptada a los nuevos formatos y contextos que demandan los jóvenes.

\section{Reconocimiento de investigación}

Los resultados de este artículo forman parte de las actividades de investigación promovidas a través del proyecto del Programa estatal de Fomento de la Investigación Científica y Técnica de Excelencia, subprograma estatal de Generación de Conocimiento del Ministerio de Economía y Competitividad de España sobre "Indicadores de gobernanza, financiación, rendición de cuentas, innovación, calidad y servicio público de las RTV europeas aplicables a España en el contexto digital" (Referencia CSO201566543-P).

\section{Bibliografía}

- Bonaut Iriarte, J. \& Vicent Ibáñez, M. (2019). Sport Content in Spanish Television Programming (1993-2010): an analysis from the audience ratings perspective. Communication \& Society, 32(3), 93- 108.

- Campos-Freire, F. (2017). El valor social de la TV abierta. Televisión Abierta. Situación actual y tendencias de la TDT, 147-166.

- EBU (2014). Public Service Values. Editorial Principles and Guidelines. www.ebuc.ch/mis. 


\section{Las nuevas narrativas, en el entorno social Universidad de La Laguna, diciembre de 2019}

- Fabbro, G y Sánchez - Labella Martín, I. (2016). Infancia, dibujos animados y televisión pública. La difusión de valores y contravalores en la producción española y argentina. Revista Mediterránea de Comunicación, 7(1), 11-29. Disponible en http://mediterraneacomunicacion.org/. DOI: 10.14198/MEDCOM2016.7.1.1

- Fishman, M. (1980). Manufacturing the news. Austin, Texas: University of Texas Press.

- García Matilla, A. (2003). ¿Es necesaria una televisión para la educación? Red Digital, N. 4., Ministerio de Educación.

- Izquierdo, J. (2016). Teoría de programación de radio y televisión. Obtenido de Universitat Jaume I: www. tenda. uji. es.

- Marco general de los medios en España (2019). Asociación para la Investigación de medios de comunicación. Recuperado el 3 de noviembre en: https://www.aimc.es/

- Maroto González, I. y Rodríguez Martelo, T. (2018). Estrategias de desarrollo de la televisión pública española: el caso de la plataforma Playz. La Innovación de la Innovación: Del Medio al Contenido Predictivo Actas del III Simposio Internacional sobre Gestión de la Comunicación, XESCOM, (A Coruña, 2018)

- Objetivos de desarrollo sostenible. (Sin fecha). En Wikipedia. Recuperado el 4 de noviembre de 2019 de https://es.wikipedia.org/wiki/Objetivos_de_Desarrollo_Sostenible

- Objetivos y metas de desarrollo sostenible (2019). En Naciones Unidas. Recuperado el 4 de noviembre de 2019 en https://www.un.org/sustainabledevelopment/es/objetivos-de-desarrollosostenible/ 


\section{Las nuevas narrativas, en el entorno social Universidad de La Laguna, diciembre de 2019}

- Rodríguez Escámez, A. (2005). Los efectos de la televisión en niños y adolescentes. Comunicar, (25),. [fecha de Consulta 1 de Noviembre de 2019]. ISSN: 1134-3478. Disponible en: https://www.redalyc.org/articulo.oa?id=158/15825053

- Sierra, V. D., \& Pascual, M. (2012). Responsabilidad Social Corporativa en el sector de la televisión. aDResearch ESIC: International Journal of Communication Research/Revista Internacional de Investigación en Comunicación, 6(6).

- Tortajada y Willem, (2019) EMPIRIA. Revista de Metodología de Ciencias Sociales. N. 42 enero-abril, 2019, pp. 99-112. ISSN: 11395737, DOI:/empiria.42.2019.23252 